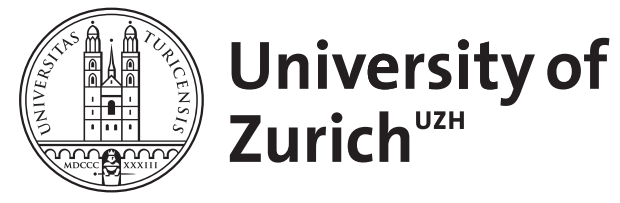

\title{
Some remarks on Liouville type theorems
}

Brezis, H ; Chipot, M ; Xie, Y

DOI: https://doi.org/10.1142/9789812709257_0003

Posted at the Zurich Open Repository and Archive, University of Zurich ZORA URL: https://doi.org/10.5167/uzh-12642

Book Section

Originally published at:

Brezis, H; Chipot, M; Xie, Y (2008). Some remarks on Liouville type theorems. In: Chipot, M. Recent advances in nonlinear analysis. Hackensack: World Scientific, 43-65.

DOI: https://doi.org/10.1142/9789812709257_0003 
MR2410737 (2010j:35057) 35B53 35B40 35J15

Brezis, H. (F-PARIS6-N); Chipot, M. [Chipot, Michel] (CH-ZRCH-DAM);

Xie, Y. [Xie, Yitian] (CH-ZRCH-DAM)

Some remarks on Liouville type theorems. (English summary)

Recent advances in nonlinear analysis, 43-65, World Sci. Publ., Hackensack, NJ, 2008.

The authors present here elementary proofs of statements related to the Liouville theorem for the equation

$$
-\nabla \cdot(A(x) \nabla u(x))+a(x) u=0
$$

in $\mathcal{D}^{\prime}\left(\mathbb{R}^{k}\right)$, where $a \in L_{\text {loc }}^{\infty}\left(\mathbb{R}^{k}\right), a(x) \geq 0$ and $A(x)=\left(a_{i j}(x)\right)$ is a $k \times k$ uniformly elliptic matrix of bounded measurable coefficients. When $A(x) \equiv \delta_{i j}$ the equation is the so-called stationary Schrödinger equation [see Y. Pinchover, in Spectral theory and mathematical physics: a Festschrift in honor of Barry Simon's 60th birthday, 329355, Proc. Sympos. Pure Math., 76, Part 1, Amer. Math. Soc., Providence, RI, 2007; MR2310209 (2008e:35002); B. Simon, Bull. Amer. Math. Soc. (N.S.) 7 (1982), no. 3, 447-526; MR0670130 (86b:81001a)]. When $a \equiv 0$ it is well known that every bounded solution has to be constant (see [L. C. Evans, Partial differential equations, Amer. Math. Soc., Providence, RI, 1998; MR1625845 (99e:35001); M. Meier, Manuscripta Math. 29 (1979), no. 2-4, 207-228; MR0545042 (80m:35024); J. Moser, Comm. Pure Appl. Math. 14 (1961), 577-591; MR0159138 (28 \#2356)] and also [H. Berestycki, I. Capuzzo Dolcetta and L. Nirenberg, Topol. Methods Nonlinear Anal. 4 (1994), no. 1, 59-78; MR1321809 (96d:35041); M. Rigoli and A. G. Setti, NoDEA Nonlinear Differential Equations Appl. 9 (2002), no. 1, 15-36; MR1891293 (2002k:35096)] for some nonlinear versions). The case where $a \neq 0$ and $k \geq 3$ is very different and in this case nontrivial bounded solutions might exist. Many of the results in this paper are known in one form or another [see S. Agmon, in Differential equations (Birmingham, Ala., 1983), 7-17, North-Holland, Amsterdam, 1984; MR0799327 (87a:35060); C. J. K. Batty, Math. Ann. 292 (1992), no. 3, 457-492; MR1152946 (93g:47050); W. Arendt, C. J. K. Batty and P. Bénilan, Math. Z. 209 (1992), no. 4, 511-518; MR1156433 (93i:47057); A. A. Grigor'yan, Trudy Sem. Petrovsk. No. 14 (1989), 66-77, 265-266; MR1001354 (90m:35050); Bull. Amer. Math. Soc. (N.S.) 36 (1999), no. 2, 135-249; MR1659871 (99k:58195); A. A. Grigor'yan and W. Hansen, Math. Ann. 312 (1998), no. 4, 659716; MR1660247 (2000a:58092); Y. Pinchover, Differential Integral Equations 5 (1992), no. 3, 481-493; MR1157482 (93b:35035); R. G. Pinsky, Positive harmonic functions and diffusion, Cambridge Univ. Press, Cambridge, 1995; MR1326606 (96m:60179); Trans. Amer. Math. Soc. 360 (2008), no. 12, 6545-6554; MR2434298 (2009i:35058)], but the proofs presented here are based on simple self-contained PDE techniques. For instance, Liouville-type results are proved in the case where the growth of $u$ is controlled (i.e., for $r$ large $r^{-2} \int_{r \Omega \backslash \frac{r}{2} \Omega} u^{2} d x \leq C$, with $\Omega$ being a bounded domain containing the origin), in the case of decay of $a(x)$ (i.e., for $|x|$ large $a(x) \geq \frac{c}{|x|^{\beta}}, \beta<2$ or $\beta=2$ and in such a case $a_{i j} \in C^{1}\left(\mathbb{R}^{k} \backslash B(0, R)\right)$ with $\partial_{i}\left(a_{i j}(x)\right) \cdot x_{j} \leq D$ for $|x|>R$ and some $\left.R>0\right)$ and in the case where at infinity $a$ has enough mass locally (i.e., $a(x) \geq a_{0}>0$ at infinity or $a(x) \geq$ $a_{p}$ where $a_{p}$ is a periodic function). When $A_{i j} \equiv \delta_{i j}$ bounded nontrivial solutions exist if $a \neq 0$ and $\int_{|x|>1} a(x)|x|^{-k+2} d x<\infty$ for $k \geq 3$, and do not exist if $a(x) \geq \bar{a}(|x|)$ for $|x|$ large with $\int^{\infty} r \bar{a}(r) d r=+\infty$. 
\{For the entire collection see MR2416199 (2009f:35002)\}

Luisa Moschini

(C) Copyright American Mathematical Society 2010, 2015 